\title{
Expanding the use of CTS-to-Self mechanism to improving broadcasting on IEEE 802.11 networks
}

\author{
Christos Chousidis, Rajagopal Nilavalan \\ School of Engineering and Design \\ Brunel University \\ London, UK \\ \{christos.chousidis, rajagopal.nilavalan\}@brunel.ac.uk
}

\author{
Laurentiu Lipan \\ Dept. of Electric Power Systems \\ University Politehnica of Bucharest \\ Bucharest, Romania \\ laurentiulipan@gmail.com
}

\begin{abstract}
The growing need for multimedia applications within wireless Local Area Networks (LAN) demands reliable and efficient broadcasting and multicasting transmission of time sensitive data, like audio and video. IEEE 802.11 standard which is the primary technology in wireless LANs was not initially designed to handle this type of data traffic. However, this raises a series of problems mainly related to the lack of an effective feedback mechanism for multicasting and broadcasting transmission. This inherited problem does not allow the standard to take full advantage of the bandwidth offered by its latest amendments. In this paper the expanding use of the CTS-to-Self protection mechanism is proposed in order to improve the performance of an ad-hoc network in a multiple broadcasting environment. The Medium Access Control (MAC) algorithm is appropriately modified and tested under various data traffic conditions. The simulations shows that this expanding use of CTS-to-Self mechanism can improve the performance of multimedia type data broadcasting in a wireless ad-hoc network.
\end{abstract}

Keywords-Broadcasting; CTS-to-Self; Ad-Hoc Networks

\section{INTRODUCTION}

The IEEE 802.11 standard (Wi-Fi) is the primary technology in wireless networking and its use has an exponential raise. This raise is reinforced by the wide use of mobile computing devices. In addition, there is an increasing demand on using of Wireless Local Area Networks (WLANs) in more specialized application, like real time audio and video networks [1]. Many of those applications are using ad-hoc networking practices and broadcasting or multicasting transmission in order to stream their data. Broadcasting is a good practice in media networking because it can distribute simultaneously data to multiple users. IEEE 802.11 standard supports broadcasting without any type of feedback (e.g. acknowledgment-ACK) from the recipients. Therefore, broadcasting does not provide any kind of delivery guarantee. IEEE 802.11 implements a carrier sense multiple access mechanism, with collision avoidance (CSMA/CA). However, collisions are still happening and due to the lack of ACK, are leading to lost data. CSMA/CA mechanism uses a random backoff technique to reduce the probability of collisions and fairly arbitrate the wireless medium by allocating random waiting time values from a predefined contention window (CW) to every station intended to transmit. The lack of positive ACK of a transmitted packet causes an exponential increase of the $\mathrm{CW}$ and therefore a decrease in the probability of collision. In broadcasting, as long as ACK is not implemented, the CW remains constant and in the case of saturated networks, the throughput is dramatically reduced [2]. An additional technique used by the CSMA/CA mechanism to arbitrate the medium access is the distribution of the network allocation vector (NAV) which contains information about the time that the network will be occupied by the station (STA), who recently gained access to the wireless medium. This technique demands an exchange of Request to Send, Clear to Send control messages (RTS/CTS) which again requires a unique recipient and therefore cannot be implemented in broadcasting. CTS-toSelf control message is an alternative to RTS/CTS process used in the cases were a CTS is not possible. It is broadcasted from a station with destination address its own address and lower transmission rate. This technique is strictly used as protection mechanism only for mixed-mode environments where extended rate physical (ERP-802.11g) and/or high throughput (HT-802.11n) devices coexist with legacy 802.11 technologies [3].

As long as ACK and NAV distribution is not implemented in broadcasting, saturated media broadcasting STAs are suffering from two main problems: large number of collisions and data loss due to buffer overflow caused by the excessively busy wireless medium. Our previous work in [4] and [5] shows that alternative backoff schemes can significantly improve throughput performance. However, these techniques can support only limited number of broadcasting STAs. The expanding use of CTS-to-Self control message, proposed in this paper, can be the solution in this problem. The IEEE 802.11 MAC algorithm is modified here in order to send a CTS-to-Self control message prior to every broadcasting packet, using the operational-high data rate. This allows STAs that have data to transmit to avoid unnecessary backoff attempts which cause dropped data due to buffer overflow. In addition, distributes network allocation information which helps STAs to defer transmission when the network is busy. It is also minimizes the effect of the collisions on the network, as the inevitable collisions, due to the small size of the CW, are happening mostly between CTS-to-Self packets which have significantly smaller size than data packets and therefore are lasting less time.

The remaining of this paper is organized as follows: In section II, the 802.11 MAC process is summarized and the drawbacks of random backoff algorithm in the case of multiple 
broadcasting are analyzed. In section III, the proposed modifications are thoroughly described. In Section IV, the simulation's characteristics are described and the results are presented and commented. Finally, in Section $\mathrm{V}$ the conclusions of this work are presented.

\section{ANALYSIS AND DRAWBACKS OF 802.11 MEDIUM ACCESS MECHANISM}

\section{A. Analysis of IEEE 802.11 MAC algorithm}

IEEE 802.11 MAC architecture [6] is based on two basic coordination functions, Point Coordination Function (PCF) and Distributed Coordination Function (DCF). PCF is a contention free access method which provides polling intervals to allow uncontended transmission opportunities for participating STAs. This function is not used here, firstly because it demands the use of an AP and secondly, because manufacturers have never implemented in practice. In this study the fundamental DCF contention-based access mechanism is used.

DCF function is described as follow. A STA with a packet to transmit waits for the channel to become idle. When an idle period equal to DCF Inter-Frame Space (DIFS) is detected, generates an initial Backoff time value (Fig 1). This value indicates the period that the STA has to additionally defer before transmitting. The random backoff process is the most important mechanism used in IEEE 802.11 CSMA/CA to prevent collisions. CW increases exponentially for every retransmission.

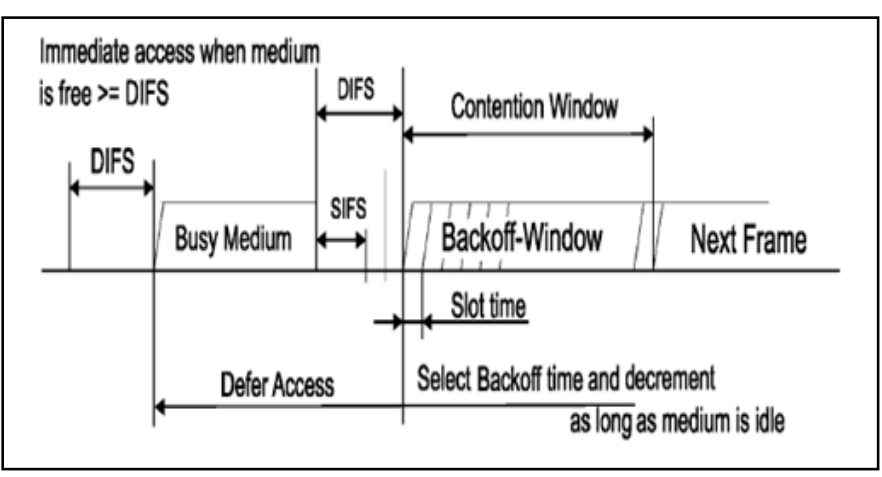

Fig 1: IEEE 802.11 basic access method

Under low utilization, stations are not forced to wait very long before transmitting their frame. If the utilization of the network is high, the protocol holds STAs back for longer period of times to minimize the probability of multiple stations transmitting at the same time. Backoff time is extracted from the following formula:

$$
\text { Backoff_Time }=\operatorname{INT}(C W \times \text { Random }(0,1)) \times \text { aSlotTime }
$$

Random $(0,1)$ is a pseudo-random number between 0 and 1 drown from a uniform distribution. $\mathrm{CW}$ is an integer within the range of values $\mathrm{CWmin}$ and $\mathrm{CWmax}$. $\mathrm{CWvalues}=2 \mathrm{x}-1$ (x starts from an integer defined by the station and goes up to 10). For example, for $\quad \mathrm{x}=4, \quad \mathrm{CW} 4=24-1=15, \quad \mathrm{CW} 5=31$, $\mathrm{CW6}=63 \ldots \mathrm{CW} 10=1023$. The aSlotTime duration is the value of the correspondingly named PHY characteristics. The Backoff timer is decremented with one slot as long as the channel is idle. When a transmission is detected, the Backoff timer freezes and start to decrease again when the channel is sensed idle for a DIFS. When the timer reaches zero the data packet is finally transmitted.

\section{B. Drawbacks of random backoff in 802.11 broadcasting}

The IEEE 802.11 standard defines that the CW size exponentially increases for each retransmission attempt of the same packet. However, as there is no retransmission in broadcasting, the $\mathrm{CW}$ size always holds the CWmin value. Under high utilization due to increasing number of STA and/or high data production, CWmin appears to be extremely small. The most significant problem in the case of multimedia multiple-broadcasting environment is that there is a high likelihood for two or more STAs to choose concurrently equal backoff value. It is easy to understand that when we have large number of STAs producing continuous data and they are performing the backoff process using a $\mathrm{CW}=15$ (like in $802.11 \mathrm{~g} \& 802.11 \mathrm{n})$ this is highly possible. In this case a collision is occurring and a data packet is lost as there is no recovery mechanism.

\section{MODIFIED MAC MECHANISM}

CTS-to-Self control message is an alternative to RTS/CTS process currently used in broadcasting only in cases where legacy technologies coexist with an ERP (802.11g) or HT (802.11n) physical, (mixed-mode networks). It is achieved by sending a CTS-to-Self control frame in appropriate (usually lower) data rate and modulation that all STAs can understand. CTS-to-Self frame contains in its "duration" field the time that all non-transmitting STAs must defer before trying to access the medium.

In order for the CTS-to-Self to be used as the main protection mechanism in broadcasting, two major modifications have been done in the 802.11 MAC algorithm. First, while the structure of the packet remained as it was described in the standard; the MAC has been reprogrammed in order to transmit a CTS-to-Self control message prior to every data transmission [7] [8]. Thus, when a STA complete its random backoff countdown and finds the medium idle, instead of broadcasting a data packet, sends a CTS-to-Self control packet which contains information concerning the time period that the medium will be reserved due to the forthcoming data packet transmission. In the unfortunate event that another STA completes the random backoff simultaneously, the two CTS-toSelf packets are colliding causing a jam in the network lasting significantly less than a data packet collision (Fig 2).

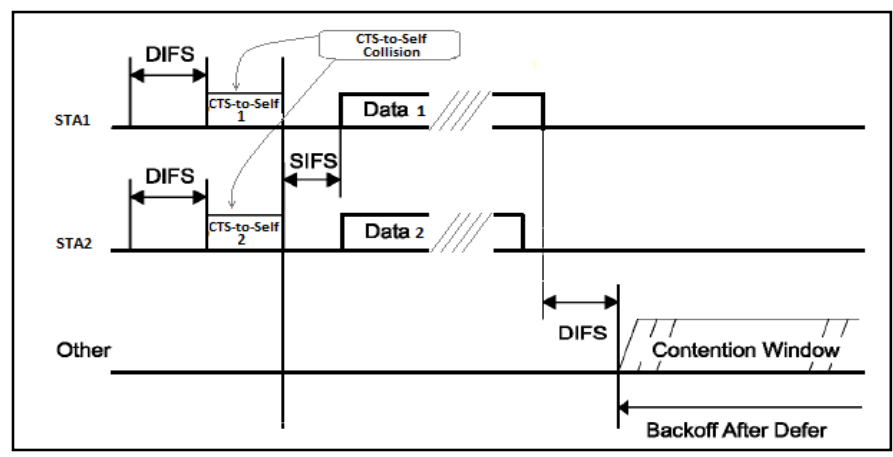

Fig 2: CTS-to-Self collision 
In any other case the STA waits for a sort inter frame period (SIFS) and transmits the data packet. The second modification in the MAC algorithm, which has been done in this project, was to reprogram the transmission rate of the CTSto-Self message. CTS-to-Self transmission parameter has been modified to always adjust with the selected data rate used for data transmission.

\section{Simulation CHARACTERISTICS AND RESUltS}

\section{A. Simulation Characteristics}

The OPNET Modeller 17.1 network simulation platform it is used in this study. The simulation is based on IEEE $802.11 \mathrm{~g}$ Physical characteristics with a bit rate of 54 Mbps. The WSTAs are forming an ad-hoc network with its population to gradually increase from 5 to 60 STAs. The packet generator in each STA is set to create a data load of $256 \mathrm{Kbps}$ which is a satisfactory average data load, produced by the most commonly used media compression codecs. The final payload in the wireless medium is found to be $320 \mathrm{Kbps}$ due to the MAC overhead. All STAs works in a saturated condition. That means that they always have packets to transmit. The generated data load remains constant in all simulations but as the number of medium access attempts for each STA depends on the packet size, three different packet sizes (2048, 1024 and 512 bytes) are used for each population increase. This allows us to test the effect of the expanding use of the CTS-to-Self protection mechanism in various traffic conditions.

\section{B. MAC Modifications}

OPNET is a powerful simulation tool which allows full access to its model's source code. The WLAN MAC process in each STA "reads" the manually set attributes and respectively invokes the appropriate "child process". In this work a modified child process is created and used together with the classic 802.11 WLAN MAC child process in order to produce comparative studies. A number of modification have been made in order to force the modified child process to create a CTS-to-Self message prior to each data packet transmission using also the operational bit rate which in this case is 54 Mbps.

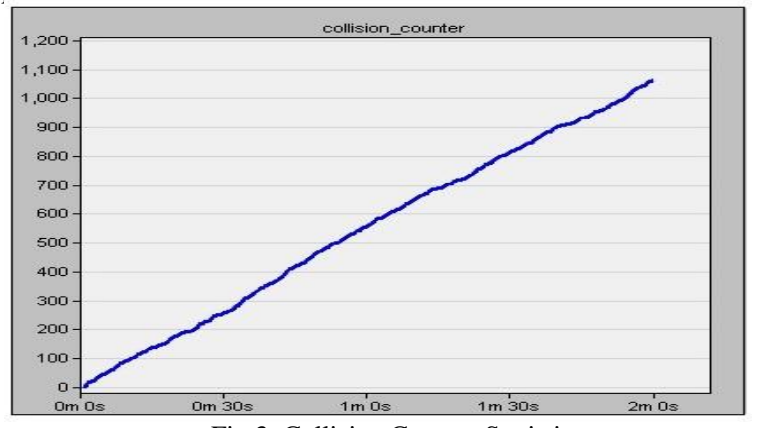

Fig 3: Collision Counter Statistic

OPNET provides a statistic which reports the collision status but there is no collective statistic to measure the number of collisions in each STA. Collisions can be measured only through "packet info report" and specifically counting the destroyed packets in the WLAN port of the receiver (wlan_port_rxO). For more accurate measurements a Collision
Counter statistic was created in both child processes. This statistic monitors the changes in the "collision flag" and precisely report the exact number of collisions encountered in each STA (Fig 3). Table I contains the settings for all different packet size simulations.

TABLE I. SimUlation SetTings

\begin{tabular}{|l|l|l|l|}
\hline $\begin{array}{l}\text { Packet } \\
\text { Size (bytes) }\end{array}$ & \multicolumn{1}{|c|}{$\mathbf{2 0 4 8}$} & \multicolumn{1}{|c|}{$\mathbf{1 0 2 4}$} & $\mathbf{5 1 2}$ \\
\hline Start Time & $\begin{array}{l}\text { Normal }(0.01, \\
0.0001)\end{array}$ & $\begin{array}{c}\text { Normal }(0.01, \\
0.0001)\end{array}$ & $\begin{array}{c}\text { Normal }(0.01, \\
0.0001)\end{array}$ \\
\hline On-State & Constant (120) sec & Constant $(120) \mathrm{sec}$ & Constant (120) sec \\
\hline Off-State & Constant (0) sec & Constant $(0) \mathrm{sec}$ & Constant $(0) \mathrm{sec}$ \\
\hline $\begin{array}{l}\text { Interarrival } \\
\text { Time }\end{array}$ & $\begin{array}{c}\text { Constant }(0.05) \\
\mathrm{sec}\end{array}$ & Constant $(0.025) \mathrm{sec}$ & Constant $(0.0125) \mathrm{sec}$ \\
\hline
\end{tabular}

\section{Simulation Results}

The simulation runs for 2 minutes. This is enough time to reach a steady state where accurate measurements can be taken. The statistics collected during the simulation are; Throughput, Overall End to End Delay and the Number of Collisions encountered in each STA. For each increase of the population a separate simulation is performed. In order to ensure accuracy, each simulation runs for three times using a different "seed" number. The final results are the average values from the three simulations.

a. Throughput

The following graphs are illustrating the throughput performance of the network, for different data packet sizes, with and without the use of CTS-to-Self as a protection mechanism.

It is clearly shown that using the proposed CTS-to-Self protection technique, we achieve better throughput performance. However, the improvement is bigger when large packets are used (Fig 4). In addition, when 512 bytes packet size is implemented, the modified MAC is the first one which collapses as expected, due to extremely large number of packets flooding the network (Fig 6).

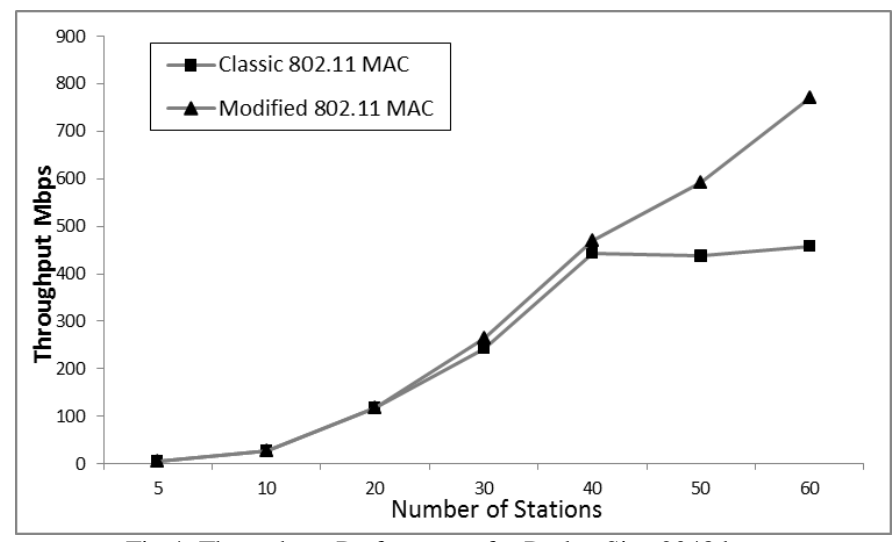

Fig 4: Throughput Performance for Packet Size 2048 bytes 


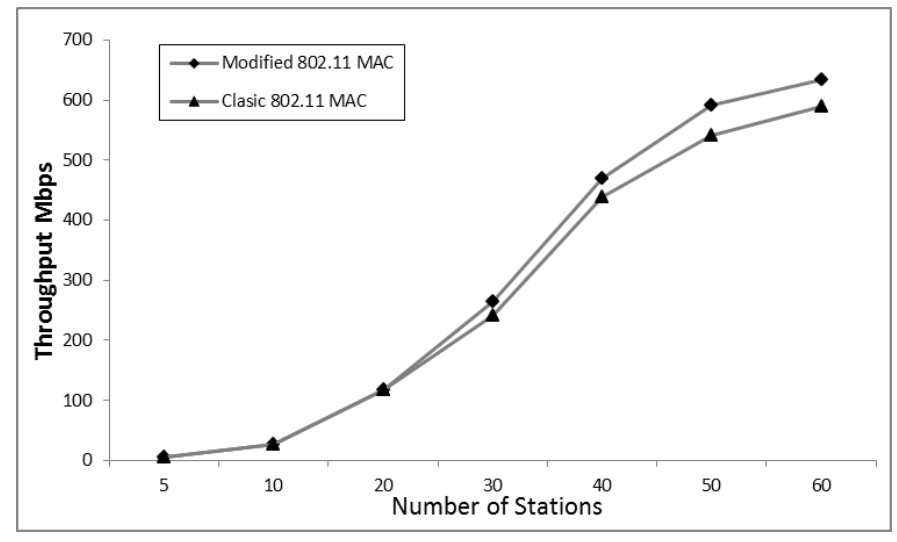

Fig 5: Throughput Performance for Packet Size 1024 bytes

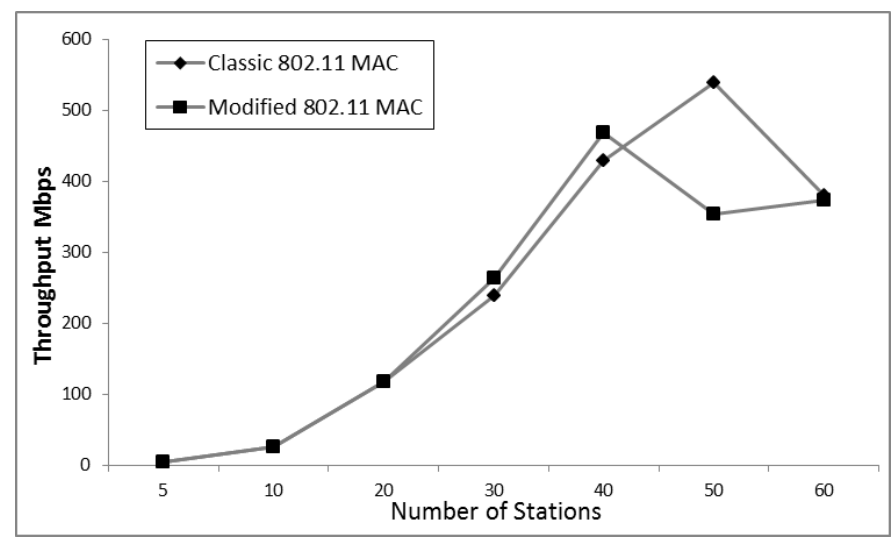

Fig 6: Throughput Performance for Packet Size 512 bytes

b. End-to-End Delay

The following graphs are illustrating the End to End Delay of all packets received by the wireless LAN MACs of all WLAN nodes in the network and forwarded to the higher layer.

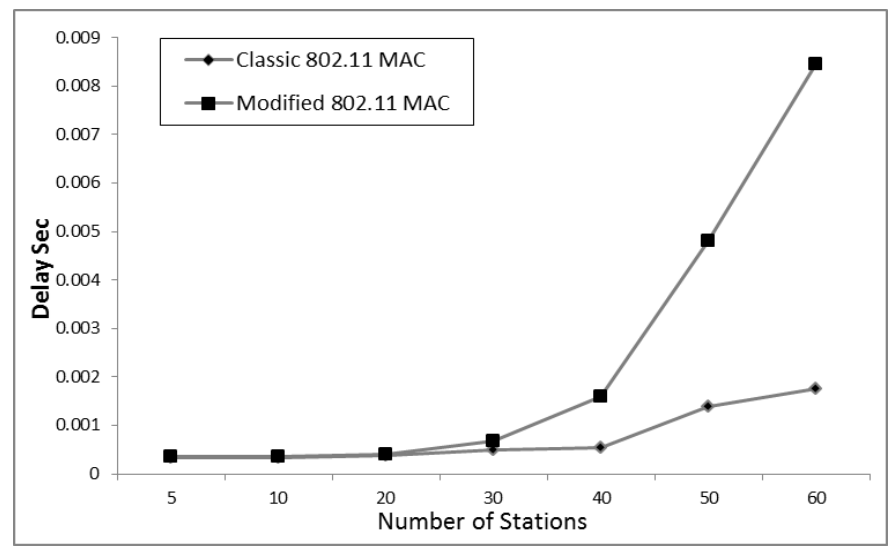

Fig 7: End-to-End Delay for Packet Size 2048 bytes

It is also shown in this study that the use of large packets gives the best performance when it comes to delay (Fig 7). As it is expected, the delay reaches higher values due to the additional CTS-to-Self transactions however remains in acceptable levels for real time media application. Decreasing packet size, the delay takes significantly higher values which in the case of
512 byte packet size are inappropriate for multimedia applications (Fig 9). In figures 8 and 9 a logarithmic scale is used for the time axis in order to illustrate the wide ranges of delay values.

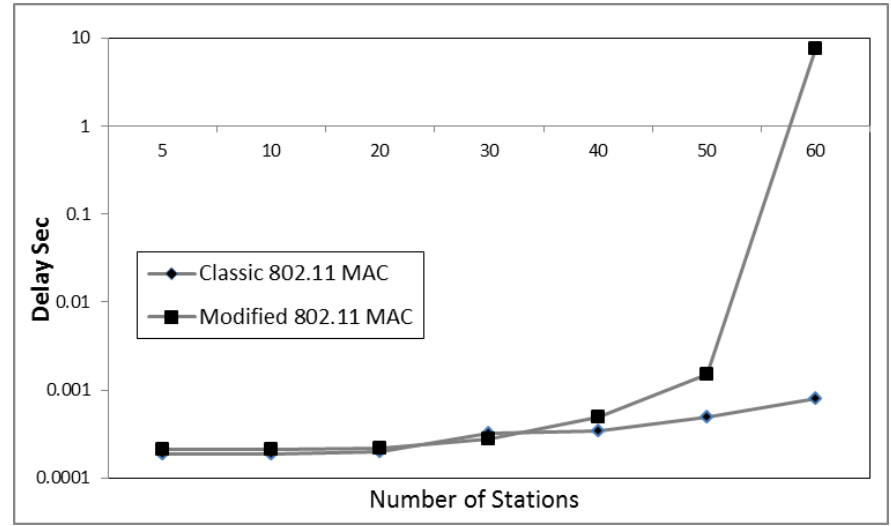

Fig 8: End-to-End Delay for Packet Size 1024 bytes

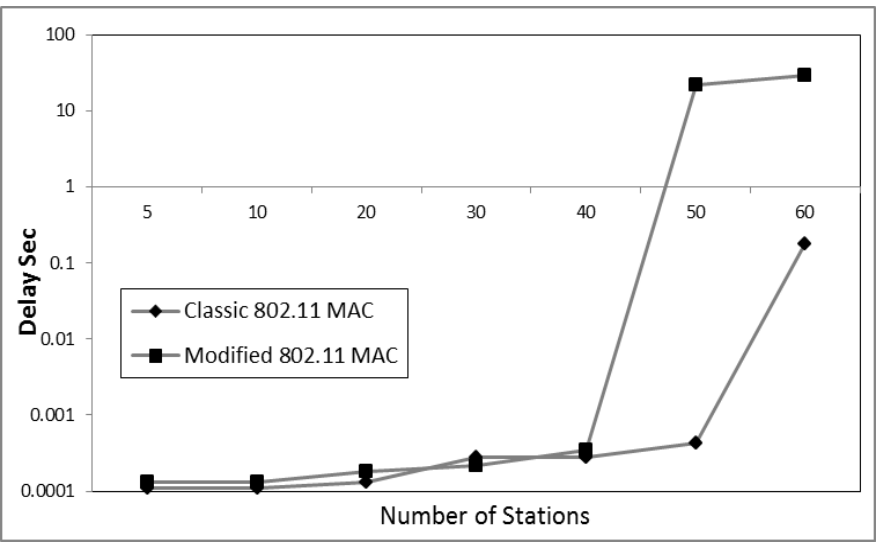

Fig 9: End-to-End Delay for Packet Size 512 bytes

c. Average Number of Collisions

Finally, it is interesting to examine the collision occurrence in the network. As we can see from figures 10, 11 and 12, although the number of collisions per STA is higher, the modified 802.11 MAC using the CTS-to-Self mechanism gives better throughput. This phenomenon is thoroughly explained in section III.

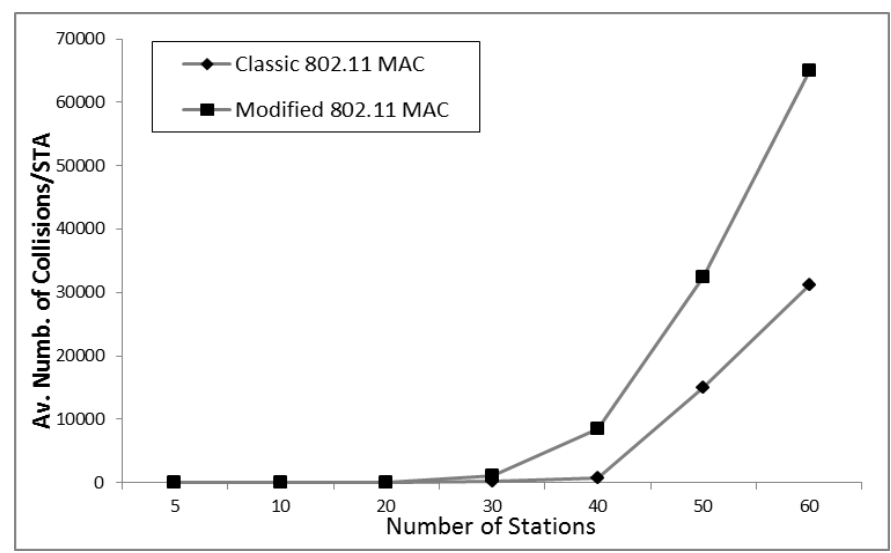

Fig 10: Average Number of Collisions per STA for Packet Size 2048 bytes 


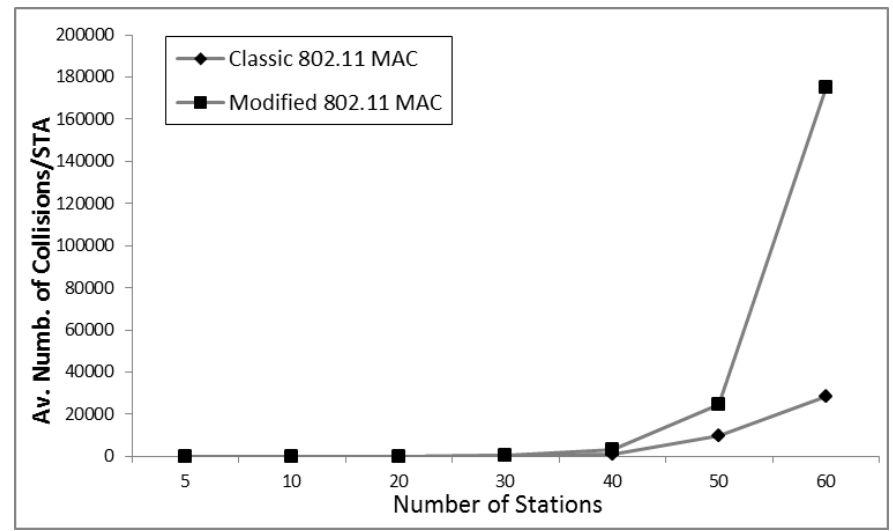

Fig 11: Average Number of Collisions per STA for Packet Size 1024 bytes

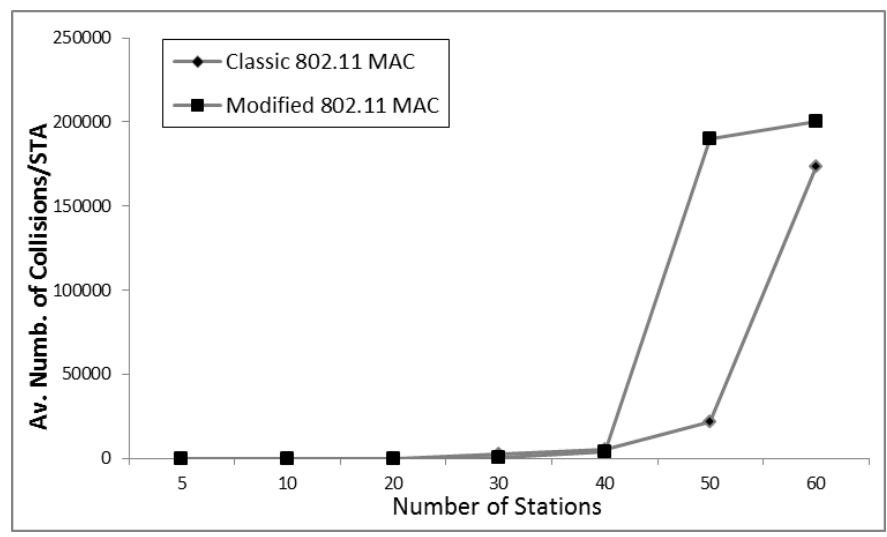

Fig 12: Average Number of Collisions per STA for Packet Size 512 bytes

\section{CONCLUSIONS}

In this paper we are investigating the possibility of improving the performance of broadcasting in a saturated IEEE 802.11 ad-hoc network using the CTS-to-Self protection mechanism. CTS-to-Self is an alternative to CTS/RTS technique used by the 802.11 standard only for mixed-mode environments where ERP and HT devices coexist with legacy 802.11 technologies. Ti has the regular CTS packet format and it is sent by a STA with destination address its own address.
We extend this idea using this control message in a heavy traffic broadcasting environment where no other protection mechanism can be used. The target is to distribute network allocation information and at the same time to limit the effect of collisions in the network. For this reason the classic 802.11 MAC is modified and various types of data traffic is applied using different packet size.

As we can see from the simulation results, this technique can significantly improve performance when many saturated STAs are broadcasting in an ad-hoc network. However this improvement can be achieved when large size packets are used. When packet size becomes small the number of packets needed to maintain the same bit rate increases. In this case, the additional traffic caused by the CTS-to-Self messages reduces the performance of the network.

Our future research will focuses on the design of an adoptive algorithm that will be able to monitor the broadcasting traffic in the network and appropriately adjusts the length of packets to the optimum size.

\section{REFERENCES}

[1] AES White Paper: Best Practices in Network Audio, JAES Volume 57 Issue 9 pp. 729-741; September 2009.

[2] A. Floros T. Karoudakis, Delivering high-quality audio over WLANs, AES 116th Convention, 2004.

[3] Fluke networks White Paper, Ensuring 802.11n snd $802.11 \mathrm{a} / \mathrm{b} / \mathrm{g}$ Compatibility, 2011.

[4] C Chousidis, R Nilavalan, A Floros, Enhancement of IEEE 802.11 in Handling Multiple Broadcasting Audio Data in Wireless ad-hoc Networks, Journal of the Audio Engineering Society 61 (4), 165-173.

[5] C Chousidis, $\mathrm{R}$ Nilavalan, Improving multiple broadcasting of multimedia traffic in wireless ad-hoc networks, Science and Information Conference, October 2013, London, UK (Accepted).

[6] IEEE Standard, Wireless LAN Medium Access Control (MAC) and Physical Layer (PHY) Specifications, 2007 M. Young, the Technical Writer's Handbook. Mill Valley, CA: University Science, 1989.

[7] Brad Williams Tracy Camp, Comparison of Broadcasting Techniques for Mobile Ad Hoc Networks, 3rd ACM international symposium on Mobile ad hoc networking \& computing, 2002. 